\title{
Gravitating defects of codimension-two
}

\author{
Georgios Kofinas* and Theodore N. Tomaras ${ }^{\dagger}$ \\ Department of Physics and Institute of Plasma Physics, University of Crete, 71003 Heraklion, Greece
}

(Dated: September 11, 2018)

\begin{abstract}
Thin gravitating defects with conical singularities in higher codimensions and with generalized Israel matching conditions are known to be inconsistent for generic energy-momentum. A way to remove this inconsistency is proposed and is realized for an axially symmetric gravitating codimensiontwo defect in six dimensional Einstein gravity. By varying with respect to the brane embedding fields, alternative matching conditions are derived, which are generalizations of the Nambu-Goto equations of motion of the defect, consistent with bulk gravity. For a maximally symmetric defect the standard picture is recovered. The four-dimensional perfect fluid cosmology coincides with conventional FRW in the case of radiation, but for dust it has $\rho^{4 / 3}$ instead of $\rho$. A four-dimensional black hole solution is presented having the Schwarzschild form with a short-distance $r^{-2}$ correction.
\end{abstract}

The study of higher codimension gravitating defects is important for many reasons. They arise naturally in higher dimensional gravity theories such as 10dimensional supergravity or M-theory, they are relevant to the discussion of braneworld models of particle physics and cosmology, while the well-known cosmic strings of four-dimensional GUTs are examples of such codimension-two objects. In particular, the codimensiontwo braneworlds in six-dimensional bulk have also attracted considerable interest recently in relation to the cosmological constant problem [1].

Here, we shall focus on the study of codimension-two gravitating defects with zero thickness. They are interesting on their own right as descriptions of D-braneworld models, and furthermore, they should be good approximations of model-dependent thick defects away from their cores 2]. However, in the standard treatment, one varies the action with respect to the metric; this leads to bulk Einstein equations with the localized brane energymomentum tensor as source proportional to $\delta^{(2)}$ on the right-hand side, which are known to be inconsistent for generic energy-momentum on the defect [3]. In this paper instead, to obtain the embedding conditions of the defect, we shall vary the action with respect to the brane position in a way that takes into account the gravitational back-reaction. Our approach is reminiscent of the "Dirac style" variation performed in [4] in the study of codimension-one defects.

We consider the total brane-bulk action

$$
\begin{aligned}
S= & \frac{1}{2 \kappa_{6}^{2}} \int_{M} d^{6} x \sqrt{|g|}\left(\mathcal{R}-2 \Lambda_{6}\right)+\int_{\Sigma} d^{4} x \sqrt{|h|}\left(\frac{r_{c}^{2}}{2 \kappa_{6}^{2}} R-\lambda\right) \\
& +\int_{M} d^{6} x \mathcal{L}_{m a t}+\int_{\Sigma} d^{4} x L_{m a t}
\end{aligned}
$$

where $g_{\mu \nu}$ is the bulk metric tensor, $h_{\mu \nu}$ is the induced metric on the brane $(\mu, \nu, \ldots$ are six-dimensional coordinate indices), $\mathcal{R}$ and $R$ are the bulk and brane scalars, $\lambda$ is

\footnotetext{
*gkofin@phys.uoa.gr

†tomaras@physics.uoc.gr
}

the brane tension and $r_{c}$ is the induced-gravity crossover length scale. $\mathcal{L}_{\text {mat }}, L_{\text {mat }}$ are the matter Lagrangians of the bulk and of the brane. The bulk equations are

$$
\mathcal{G}_{\mu \nu}=\kappa_{6}^{2} \mathcal{T}_{\mu \nu}-\Lambda_{6} g_{\mu \nu}
$$

where $\mathcal{G}_{\mu \nu}$ is the bulk Einstein tensor and $\mathcal{T}_{\mu \nu}$ is a regular bulk energy-momentum tensor. In addition, we consider $n_{\alpha}{ }^{\mu}(\alpha=1,2)$ arbitrary unit vectors normal to the brane. The induced metric $h_{\mu \nu}$ is given by $h_{\mu \nu}=g_{\mu \nu}-n_{\alpha \mu} n^{\alpha}{ }_{\nu}$, or equivalently, in the basis $\left(\partial_{i}, n_{\alpha}\right)$ by $h_{i j}=g_{\mu \nu} x_{, i}^{\mu} x_{, j}$ $(i, j, \ldots$ are coordinate indices on the brane).

The ordinary way to consider the interaction of the brane with the bulk is through the variation $\delta g_{\mu \nu}$ at the position of the brane, in which case one should add on the right-hand side of equation (2) the term $\kappa_{6}^{2} \tilde{T}_{\mu \nu} \delta^{(2)}$, where $\tilde{T}_{\mu \nu}=T_{\mu \nu}-\lambda h_{\mu \nu}-\left(r_{c}^{2} / \kappa_{6}^{2}\right) G_{\mu \nu} . T_{\mu \nu}$ is the brane energy-momentum tensor, $G_{\mu \nu}$ the brane Einstein tensor and $\delta^{(2)}$ the two-dimensional delta function. The presence of $\delta^{(2)}$ in the resulting equation leads to matching conditions from the distributional terms with the known inconsistencies.

Here, we propose that the interaction of the brane with the bulk is obtained by varying the action with respect to $\delta x^{\mu}$, the embedding fields of the brane position. In this case, $\delta g_{\mu \nu}=-£_{\delta x} g_{\mu \nu} . \delta h_{i j}$ and $\delta n_{\alpha \mu}$ could also be expressed in terms of $\delta x^{\mu}$. However, we find it more convenient to vary $h_{i j}$ and $n_{\alpha \mu}$ independently by including corresponding Lagrange multipliers. So, the relation of $h_{i j}$ to $g_{\mu \nu}$ and the orthonormality of $n_{\alpha}{ }^{\mu}$ require the addition to $S$ of the following constraint action

$$
\begin{aligned}
S_{c}=\int_{\Sigma} d^{4} x & \sqrt{|h|}\left[\lambda^{i j}\left(h_{i j}-g_{\mu \nu} x_{, i}^{\mu} x_{, j}^{\nu}\right)\right. \\
& \left.+\lambda^{\alpha i} n_{\alpha \mu} x_{, i}^{\mu}+\lambda^{\alpha \beta}\left(g_{\mu \nu} n_{\alpha}{ }^{\mu} n_{\beta}{ }^{\nu}-\delta_{\alpha \beta}\right)\right],
\end{aligned}
$$

where $\lambda^{i j}, \lambda^{\alpha i}, \lambda^{\alpha \beta}$ are Lagrange multipliers. Variation of $S+S_{c}$ with respect to $n_{\alpha \mu}, h_{i j}, g_{\mu \nu}$ gives

$$
\begin{aligned}
& \left.\delta\left(S+S_{c}\right)\right|_{\text {brame }}=\int_{\Sigma} d^{4} x \sqrt{|h|}\left(\lambda^{\alpha i} x_{, i}^{\mu}+2 \lambda^{\alpha \beta} n_{\beta}{ }^{\mu}\right) \delta n_{\alpha \mu} \\
& +\int_{\Sigma} d^{4} x \sqrt{|h|}\left[\lambda^{i j}+\frac{1}{2}\left(T^{i j}-\lambda h^{i j}\right)-\frac{r_{c}^{2}}{2 \kappa_{6}^{2}} G^{i j}\right] \delta h_{i j}
\end{aligned}
$$




$$
\begin{aligned}
& +\int_{\Sigma} d^{4} x \sqrt{|h|}\left(\lambda^{\alpha \beta} n_{\alpha}{ }^{\mu} n_{\beta}{ }^{\nu}-\lambda^{i j} x_{, i}^{\mu} x_{, j}^{\nu}\right) \delta g_{\mu \nu} \\
& +\left.\frac{1}{2 \kappa_{6}^{2}} \int_{M} d^{6} x \sqrt{|g|}\left[\left(\mathcal{G}_{\mu \nu}-\kappa_{6}^{2} \mathcal{T}_{\mu \nu}\right) \delta g^{\mu \nu}+2 g^{\mu[\kappa} g^{\lambda] \nu}\left(\delta g_{\nu \kappa}\right)_{; \lambda \mu}\right]\right|_{\text {bram }}
\end{aligned}
$$

When $r_{c} \neq 0$, one should add in (11) the integral of the extrinsic curvature $k$ of $\partial \Sigma$; this, in general, does not affect the dynamics of $\Sigma[5]$.

For simplicity, we restrict ourselves to the axially symmetric bulk ansatz

$$
d s_{6}^{2}=d \varrho^{2}+L^{2}(x, \varrho) d \varphi^{2}+h_{i j}(x, \varrho) d x^{i} d x^{j} .
$$

$h_{i j}(x, 0)$ is the braneworld metric, assumed to be regular everywhere with the possible exception of isolated singular points [6]. $\varphi$ has the standard periodicity $2 \pi$ and we make the usual assumption for conical singularities $L(x, \varrho)=\beta(x) \varrho+\mathcal{O}\left(\varrho^{2}\right)$ for $\varrho \approx 0, L^{\prime}(x, 0)=1$, where a prime denotes differentiation with respect to $\varrho$. The non-vanishing components of $\mathcal{G}_{\mu \nu}$ are

$$
\begin{aligned}
& \mathcal{G}_{i j}=G_{i j}+\frac{L^{\prime \prime}}{L} h_{i j}-\frac{1}{2} h_{i j}^{\prime \prime}+\frac{1}{2} h_{i k}^{\prime} h_{j \ell}^{\prime} h^{k \ell}-\frac{1}{4} h^{k \ell} h_{k \ell}^{\prime} h_{i j}^{\prime} \\
& +\frac{1}{2} h^{k \ell} h_{k \ell}^{\prime \prime} h_{i j}+\frac{3}{8} h^{k \ell \prime} h_{k \ell}^{\prime} h_{i j}+\frac{1}{8}\left(h^{k l} h_{k l}^{\prime}\right)^{2} h_{i j} \\
& +\frac{1}{L^{2}}\left(\mathcal{R}_{\varphi i \varphi j}-\mathcal{R}_{\varphi k \varphi \ell} h^{k \ell} h_{i j}\right) \\
& \mathcal{G}_{\varrho \varrho}=-\frac{1}{2} R+\frac{1}{8} h^{k \ell \prime} h_{k \ell}^{\prime}+\frac{1}{8}\left(h^{k \ell} h_{k \ell}^{\prime}\right)^{2}-\frac{1}{L^{2}} h^{k \ell} \mathcal{R}_{\varphi k \varphi \ell} \\
& \mathcal{G}_{\varphi \varphi}=-\frac{L^{2}}{2}\left[R-h^{k \ell} h_{k \ell}^{\prime \prime}-\frac{3}{4} h^{k \ell \prime} h_{k \ell}^{\prime}-\frac{1}{4}\left(h^{k \ell} h_{k \ell}^{\prime}\right)^{2}\right] \\
& \mathcal{G}_{\varrho i}=\frac{1}{L^{2}} \mathcal{R}_{\varphi \varrho \varphi i}+h^{k \ell} \mathcal{R}_{\varrho k i \ell},
\end{aligned}
$$

where $\mathcal{R}_{\varphi i \varphi j}=L\left(L_{, k} \Gamma_{i j}^{k}-L_{, i j}-\frac{1}{2} L^{\prime} h_{i j}^{\prime}\right), \quad \mathcal{R}_{\varphi \varrho \varphi i}=$ $L\left(\frac{1}{2} L_{, k} h^{k \ell} h_{\ell i}^{\prime}-L_{, i}^{\prime}\right)$, while $\mathcal{R}_{\varrho i j k}$ does not contain $L$. Integrate over the $(\varrho, \varphi)$ transverse disc of radius $\epsilon$ the sixdimensional terms of (4) in the limit $\epsilon \rightarrow 0$. For smooth $\delta g_{\mu \nu}$, the only term in the above components of $\mathcal{G}_{\mu \nu}$ which contributes is $\left(L^{\prime \prime} / L\right) h_{i j}$, having the most singular $\delta(\varrho) / \varrho$ structure. The result is

$$
\begin{aligned}
& \left.\delta\left(S+S_{c}\right)\right|_{\text {brame }}=\int_{\Sigma} d^{4} x \sqrt{|h|}\left(\lambda^{\alpha i} x_{, i}^{\mu}+2 \lambda^{\alpha \beta} n_{\beta}{ }^{\mu}\right) \delta n_{\alpha \mu} \\
& +\int_{\Sigma} d^{4} x \sqrt{|h|}\left[\lambda^{i j}+\frac{1}{2}\left(T^{i j}-\lambda h^{i j}\right)+\frac{\pi}{\kappa_{6}^{2}}(1-\beta) h^{i j}-\frac{r_{c}^{2}}{2 \kappa_{6}^{2}} G^{i j}\right] \delta h_{i j} \\
& +\int_{\Sigma} d^{4} x \sqrt{|h|}\left(\lambda^{\alpha \beta} n_{\alpha}{ }^{\mu} n_{\beta}{ }^{\nu}-\lambda^{i j} x_{, i}^{\mu} x_{, j}{ }_{j}\right) \delta g_{\mu \nu} .
\end{aligned}
$$

At this point, had we considered $\delta g_{\mu \nu}, \delta h_{i j}, \delta n_{\alpha \mu}$ independent, all Lagrange multipliers would have been zero, leading to the matching conditions $\kappa_{6}^{2} T_{\mu \nu}=\left[\kappa_{6}^{2} \lambda-\right.$ $2 \pi(1-\beta)] h_{\mu \nu}+r_{c}^{2} G_{\mu \nu}$, which are incompatible with any $T_{\mu \nu}$ other than a brane tension [7]. Instead, as explained above, our variation is with respect to $x^{\mu}$. So,

$$
\begin{aligned}
& \left.\delta\left(S+S_{c}\right)\right|_{\text {brame }}=0 \text { gives } \\
& \lambda^{\alpha i} x_{, i}^{\mu}+2 \lambda^{\alpha \beta} n_{\beta}{ }^{\mu}=0 \\
& \lambda^{i j}=\frac{1}{2 \kappa_{6}^{2}}\left\{\left[\kappa_{6}^{2} \lambda-2 \pi(1-\beta)\right] h^{i j}-\kappa_{6}^{2} T^{i j}\right\}+\frac{r_{c}^{2}}{2 \kappa_{6}^{2}} G^{i j} \\
& \int_{\Sigma} d^{4} x \sqrt{|h|}\left(\lambda^{\alpha \beta} n_{\alpha}{ }^{\mu} n_{\beta}{ }^{\nu}-\lambda^{i j} x_{, i}^{\mu} x^{\nu}{ }_{, j}\right) \delta g_{\mu \nu}=0,
\end{aligned}
$$

where $\delta g_{\mu \nu}=-£_{\delta x} g_{\mu \nu}=-\left(g_{\mu \nu, \lambda} \delta x^{\lambda}+g_{\mu \lambda} \delta x_{, \nu}^{\lambda}+g_{\nu \lambda} \delta x_{, \mu}^{\lambda}\right)$. Since $x_{, i}^{\mu}, n_{\alpha}{ }^{\mu}$ are independent, equation (11) implies $\lambda^{\alpha i}=\lambda^{\alpha \beta}=0$. Then, equation (13) with $\lambda_{i j}$ given by equation (12) takes the form

$$
\int_{\Sigma} d^{4} x \sqrt{|h|} \lambda^{i j}\left(g_{\mu \nu, \lambda} x_{, i}^{\mu} x_{, j}^{\nu} \delta x^{\lambda}+2 g_{\mu \nu} x_{, i}^{\mu} x_{, j}^{\lambda} \delta x_{, \lambda}^{\nu}\right)=0,
$$

which, after an integration by parts and imposing $\left.\delta x^{\mu}\right|_{\partial \Sigma}=0$, becomes

$$
\int_{\Sigma} d^{4} x \sqrt{|h|} g_{\mu \sigma}\left[\lambda^{i j}{ }_{\mid j} x_{, i}^{\mu}+\lambda^{i j}\left(x_{; i j}^{\mu}+\Gamma_{\nu \lambda}^{\mu} x_{, i}^{\nu} x_{, j}^{\lambda}\right)\right] \delta x^{\sigma}=0(1
$$

In (15), | and ; denote covariant differentiations corresponding to $h_{i j}$ and $g_{\mu \nu}$ respectively. Due to the arbitrariness of $\delta x^{\mu}$ and since the extrinsic curvatures of the brane $K_{\alpha i j}=n_{\alpha i ; j}$ satisfies $-K^{\alpha}{ }_{i j} n_{\alpha}{ }^{\mu}=x_{; i j}^{\mu}+$ $\Gamma_{\nu \lambda}^{\mu} x_{, i}^{\nu} x_{, j}^{\lambda}$, the last equation is equivalent to $\lambda_{\mid j}^{i j} x_{, i}{ }_{,}-$ $\lambda^{i j} K^{\alpha}{ }_{i j} n_{\alpha}^{\mu}=0$, from which $\lambda_{\mid j}^{i j}=\lambda^{i j} K^{\alpha}{ }_{i j}=0$. Finally, using (12) we obtain

$$
\begin{aligned}
& T_{\mid j}^{i j}=\frac{2 \pi}{\kappa_{6}^{2}} h^{i j} \beta_{, j} \\
& \left\{\kappa_{6}^{2} T^{i j}-\left[\kappa_{6}^{2} \lambda-2 \pi(1-\beta)\right] h^{i j}-r_{c}^{2} G^{i j}\right\} K^{\alpha}{ }_{i j}=0 .
\end{aligned}
$$

Equation (17) is the generalization of the Nambu-Goto equation of motion, when the self-gravitating brane interacts with bulk gravity. Indeed, in the special case of a probe brane of tension $\lambda$ (no back-reaction, $T_{i j}=0$, $r_{c}=0$ ), equation (17) becomes $h^{i j} K^{\alpha}{ }_{i j}=0$, or equivalently $\square_{h} x^{\mu}+\Gamma_{\nu \lambda}^{\mu} h^{\nu \lambda}=0$, which is the Nambu-Goto equation of motion [8].

We will now examine all the bulk field equations (2) at the position of the brane and check their compatibility with the matching conditions (16), (17). Focusing on the $\mathcal{O}(1 / \varrho)$ terms in the $\varrho i$ components of equations (2) (which cannot be canceled by any regular $\mathcal{T}_{\mu \nu}$ in (2) we obtain $\beta_{, i}=0$, and equation (16) gives the exact conservation on the brane

$$
T_{\mid j}^{i j}=0 \text {. }
$$

Similarly, from the $\mathcal{O}(1 / \varrho)$ part of the $\varrho \varrho$ component of (2) we obtain $h^{i j} h_{i j}^{\prime}=0$, valid at the position of the brane. The only nontrivial remaining components of (2) with a $\mathcal{O}(1 / \varrho)$ part are the $i j$ ones, which give on the brane the equation $\widehat{L^{\prime \prime}} h_{i j}+\frac{\beta}{2}\left(h^{k \ell} h_{k \ell}^{\prime} h_{i j}-h_{i j}^{\prime}\right)=0$, with a 
hat denoting the regular part of the corresponding quantity. This equation is equivalent to $\widehat{L^{\prime \prime}} h_{i j}=\frac{\beta}{2} h_{i j}^{\prime}$, which means that on the brane $h_{i j}^{\prime}=0$. Thus,

$$
K^{\alpha}{ }_{i j}=0,
$$

which trivially satisfies (17), and from which one obtains for the brane the geodesic equation $x_{; i j}^{\mu}+\Gamma_{\nu \lambda}^{\mu} x_{, i}^{\nu} x_{, j}^{\lambda}=0$.

A few comments are in order at this point: First, it may be natural to expect that in the "strong probe limit" defined as the limit $\tilde{T}_{\mu \nu} \rightarrow 0$, the brane obeys the geodesic equation. This is trivially the case with the axially symmetric codimension-two defects, as described above by the geodesic equation (19). Incidentally, the codimension-one Israel matching conditions with $Z_{2}$-symmetry in $D$-dimensions $2 K_{i j}=-\kappa_{D}^{2}\left[\tilde{T}_{i j}-\right.$ $\left.\tilde{T} h_{i j} /(D-2)\right]$ satisfy this expectation. On the contrary, the codimension-one matching condition arising by variation with respect to $x^{\mu},\left\{2 K_{i j}+\kappa_{D}^{2}\left[\tilde{T}_{i j}-\tilde{T} h_{i j} /(D-\right.\right.$ 2)] $\} K^{i j}=0$ does not. Second, the six-dimensional curvature invariants at the position of the brane contain singu$\operatorname{lar} \delta(\varrho) / \varrho$ terms dealt above, and, in general, also terms containing powers of $1 / \varrho$ multiplied by $h_{i j}^{\prime}$, which vanish because $h_{i j}^{\prime}=0$. This means that the bulk geometry is regular at the position of the brane. Third, in the general non-axially symmetric case, the bulk equations will not necessarily imply the geodesic motion for the defect and the curvature will be divergent at its position [9]. Furthermore, the corresponding matching conditions are not expected to be trivially satisfied.

Finally, we focus on the regular part of (2) on the brane. The $\varrho \varrho$ component gives

$$
R=2 \Lambda_{6}-2 \kappa_{6}^{2} \mathcal{T}_{\varrho} .
$$

Similarly, from the $\varrho i$ components one obtains $h^{k \ell} \mathcal{R}_{\varrho k i \ell}=\kappa_{6}^{2} \mathcal{T}_{\varrho i}$, or equivalently $h^{k \ell}\left(K_{\varrho k i ! \ell}-K_{\varrho k \ell ! i}\right)=$ $\kappa_{6}^{2} \mathcal{T}_{\varrho i}$. At this point, we have used the geometric identity $\mathcal{R}^{\alpha}{ }_{i j k}=K^{\alpha}{ }_{i j ! k}-K^{\alpha}{ }_{i k ! j}$, where the covariant derivative ! is defined with respect to the connection $\varpi_{\beta \alpha i}=g\left(\nabla_{i} n_{\alpha}, n_{\beta}\right)$ as $\Phi_{\beta ! i}^{\alpha}=\Phi_{\beta \mid i}^{\alpha}+\varpi_{\gamma i}^{\alpha} \Phi_{\beta}^{\gamma}-\varpi_{\beta i}^{\gamma} \Phi_{\gamma}^{\alpha}$ for fields $\Phi_{\beta}^{\alpha}$ transforming as tensors under normal frame rotations $\Phi_{\beta}^{\alpha} \rightarrow O_{\beta}{ }^{\delta}\left(O^{-1}\right)_{\gamma}^{\alpha} \Phi_{\delta}^{\gamma}$ (the derivative | in the previous expression is meant on tangential indices $i, j, \ldots$ that $\Phi_{\beta}^{\alpha}$ may possess). Therefore, due to (19), the previous $\varrho i$ equation is identically satisfied whenever $\mathcal{T}_{\varrho i}=0$ on the brane. Concerning the regular part $\varphi \varphi$ of (2), and using (19), (20) one obtains

$$
h^{i j} \widehat{h_{i j}^{\prime \prime}}=2 \kappa_{6}^{2}\left(\mathcal{T}_{\varphi}^{\varphi}-\mathcal{T}_{\varrho}^{\varrho}\right) .
$$

Finally, using (21), the $i j$ part becomes

$$
\widehat{h_{i j}^{\prime \prime}}=2 G_{i j}+2 \Lambda_{6} h_{i j}-2 \kappa_{6}^{2}\left[\mathcal{T}_{i j}-\left(\mathcal{T}_{\varphi}^{\varphi}-\mathcal{T}_{\varrho}^{\varrho}\right) h_{i j}\right] .
$$

Combining (20), (21) and (22) one gets on the brane $2 \Lambda_{6}=\kappa_{6}^{2}\left(\mathcal{T}_{i}^{i}+\mathcal{T}_{\varrho}^{\varrho}-3 \mathcal{T}_{\varphi}^{\varphi}\right)$ and

$$
R=\kappa_{6}^{2}\left(\mathcal{T}_{i}^{i}-\mathcal{T}_{\varrho}^{\varrho}-3 \mathcal{T}_{\varphi}^{\varphi}\right) .
$$

In summary, the brane-bulk system is described by the regular piece of equations (2), under the boundary conditions (18), (19) and (23). In the absence of bulk matter $\mathcal{T}_{\mu \nu}=0$, one gets $\Lambda_{6}=0$ [10], and (23) reduces to $R=0$. This Ricci scalar flat condition is enough in special cases of high symmetry to determine the brane geometry.

We now proceed with the discussion of a few cases with physical interest: The maximally symmetric solution of equation $R=0$ is the Minkowski brane. The bulk solution which is consistent with this and (19) is the locally flat geometry with a deficit angle [11] $d s_{6}^{2}=d \varrho^{2}+\beta^{2} \varrho^{2} d \varphi^{2}-d t^{2}+d \vec{x}^{2}$, where $\beta$ is an integration constant [12]. Here, we do not have equation $\mathcal{G}_{\mu \nu}=-\kappa_{6}^{2} \lambda h_{\mu \nu} \delta^{(2)}$ to obtain the standard relation $\kappa_{6}^{2} \lambda=2 \pi(1-\beta)$ [13]. The latter we would like to arise as a direct calculation of an appropriately defined energy of the gravitational field. Indeed, one such definition that has successfully passed various other tests is given by the teleparallel representation of Einstein gravity described in [14], and gives the energy per unit length of the defect of the gravitational field of our bulk solution inside a cylinder of arbitrary radius around the defect exactly equal to $(1-\beta) / 4$.

Next, we consider the bulk cosmological metric of the form $d s_{6}^{2}=d \varrho^{2}+L^{2}(t, \varrho) d \varphi^{2}-n^{2}(t, \varrho) d t^{2}+$ $a^{2}(t, \varrho) \gamma_{\hat{i} \hat{j}}(x) d x^{\hat{i}} d x^{\hat{j}}$, where $\gamma_{\hat{i} \hat{j}}$ is a maximally symmetric 3 -dimensional metric characterized by its spatial curvature $k=-1,0,1$. For this metric, equation $R=0$ is $n^{-1} \dot{H}+2 H^{2}+k a^{-2}=0$, with $H=\dot{a} / n a$ being the Hubble parameter of the brane and a dot denotes differentiation with respect to $t$. The lapse function $n(t, 0)$ can be left undetermined, since it corresponds to the temporal gauge choice. The matter on the brane is taken to be a perfect fluid with energy density $\rho$ and pressure $p=w \rho$, in which case, the conservation equation (18) gives $\rho=M a^{-3(1+w)}$, with $M$ an integration constant. The equation for $H$ is integrated to $H^{2}=c_{1} a^{-4}-k a^{-2}$, with $c_{1}$ integration constant, or writing in terms of $\rho$, $H^{2}=c \rho^{4 / 3(1+w)}-k a^{-2}$, with $c=c_{1} M^{-4 / 3(1+w)}$. Since in the matter era the Hubble parameter falls faster than in the standard scenario, the age of the universe is predicted to be the $3 / 4$ of the one given in the standard cosmology. Moreover, since the radiation era is left unchanged, the hot big bang predictions remain intact. Note that a nonvanishing $\mathcal{T}_{\mu \nu}$ would give a contribution to the effective cosmological constant, that could drive an inflationary or a late-time acceleration era. Indeed, curiously enough, the fitting [15] to the supernovae data of the previous Hubble evolution gave $\chi^{2}=175$ (about $1 \sigma$ better than in $\Lambda \mathrm{CDM}$ ) and $\Omega_{m}=0.17$. However, a full six-dimensional treatment would be needed to study perturbative aspects of this cosmology, such as CMB or structure formation.

Finally, we consider a static spherically symmetric brane in the six dimensional bulk of the form $d s_{6}^{2}=$ $d \varrho^{2}+L^{2}(r, \varrho) d \varphi^{2}-A(r, \varrho) d t^{2}+B(r, \varrho) d r^{2}+C(r, \varrho) d \Omega_{2}^{2}$, where we can use the gauge choice on the brane $C(r, 0)=r^{2}$ to bring the four-dimensional metric in the standard form. There are two functions to be determined on 
the brane: $A(r, 0), B(r, 0)$. However, the $R=0$ equation is not enough to determine both. To close the system, consider as an example the ansatz on the brane $B(r, 0)=A(r, 0)^{-1}$. Then, $A(r)=A(r, 0)$ satisfies $r^{2} A^{\prime \prime}+4 r A^{\prime}+2 A-2=0$, with the general solution $A(r)=1+c_{1} r^{-1}+c_{2} r^{-2}\left(c_{1}, c_{2}\right.$ integration constants). This is the standard Schwarzschild metric with a short distance $r^{-2}$ correction term.

In analogy with the present treatment of axial symmetry, the addition of a Gauss-Bonnet term in the bulk [16] would lead to the resulting matching condition

$$
\begin{aligned}
& \left\{K_{\ell}^{\beta \ell} K_{\beta i j}-K_{i}^{\beta \ell} K_{\beta j \ell}+\frac{1}{2}\left(K^{\beta k \ell} K_{\beta k \ell}-K_{k}^{\beta k} K_{\beta}{ }_{\ell}\right) h_{i j}\right. \\
& \left.+\left(\beta^{-1}-1+\frac{r_{c}^{2}}{8 \pi \alpha \beta}\right) G_{i j}+\frac{\kappa_{6}^{2} \lambda-2 \pi(1-\beta)}{8 \pi \alpha \beta} h_{i j}-\frac{\kappa_{6}^{2}}{8 \pi \alpha \beta} T_{i j}\right\} K^{\alpha i j}=0,
\end{aligned}
$$

obtained under the assumptions of discontinuous $L^{\prime}$ and $h_{i j}^{\prime}\left(h_{i j}^{\prime}(x, 0)=0 \neq h_{i j}^{\prime}\left(x, 0^{+}\right)\right)$. In this case, $\beta$ in general will not be constant, and equation (19) will not be true any longer, which means that the matching condition is not trivially satisfied. By counting, however, the degrees of freedom and the equations we find an agreement, thus, we still expect that the whole bulk system will be compatible with this new matching condition leading to a final complicated braneworld dynamics.

To summarize, we studied a codimension-two zerothickness brane in the context of six-dimensional Einstein gravity, possibly extended by the addition of an induced gravity term on the brane. Our discussion focused on the axially symmetric bulk geometry.

It is well known that the analogue of the Israel matching conditions of such braneworlds (obtained by varying the action with respect to the brane metric) are inconsistent for any matter content on the brane, with the exception of just brane tension. Here, alternative matching conditions for the brane are proposed, obtained instead, by varying the action with respect to the brane position. They are generalizations of the Nambu-Goto equations of motion of a probe brane, to take into account the gravitational back-reaction. They are compatible with the full set of field equations at the position of the brane, and according to these, the brane motion in the bulk is geodesic.

The geometry on the brane itself is constrained by a vanishing induced Ricci scalar, plus the standard energymomentum conservation equation on the brane. In simple cases of high symmetry these conditions are enough to determine the geometry. We analyzed the following such characteristic cases of physical interest. For a maximally symmetric defect the standard picture of a locally flat geometry with the right deficit angle is recovered. For an isotropic perfect fluid four-dimensional cosmology, the Friedmann equation derived coincides with the conventional cosmology in the radiation era, while in the matter-dominated era it behaves like $\rho^{4 / 3}$ instead of $\rho$. For a four-dimensional spherically symmetric braneworld with the ansatz $g_{t t}=g_{r r}^{-1}$, the geometry on the brane is the Schwarzschild one with a short distance $r^{-2}$ correction term.

Extending the present analysis to a generic - less symmetric - bulk is expected to lead to more complicated dynamics, able to address also the issue of a fluctuating defect. Generalization to other gravitational theories and study of even higher-codimension defects of string theory would be extensions of the present work.

Acknowlegements. We wish to thank C. Charmousis, A. Davidson, V. Frolov, R. Maartens, G. Panotopoulos, L. Perivolaropoulos and A. Petkou for useful discussions.
[1] For a review see C. Burgess, Ann. Phys. 313 (2004) 283 hep-th/0402200; hep-th/0510123.

[2] B. Boisseau, C. Charmousis and B. Linet, Phys. Rev. D55 (1997) 616 gr-qc/9607029; K. Nakamura, A. Ishibashi and H. Ishihara, Phys. Rev. D62 (2000) 101502 gr-qc/0001015; R. Battye, B. Carter and A. Mennim, Phys. Rev. D71 (2005) 104026 hep-th/0412053.

[3] W. Israel, Phys. Rev. D15 (1977) 935; R. Geroch and J. Traschen, Phys. Rev. D36 (1987) 1017; D. Garfinkle, Class. Quant. Grav. 16 (1999) 4101 gr-qc/9906053; J. Cline, J. Descheneau, M. Giovannini and J. Vinet, JHEP 0306 (2003) 048 hep-th/0304147.

[4] A. Davidson and I. Gurwich, Phys. Rev. D74 (2006) 044023 gr-qc/0606098.

[5] R. Capovilla and J. Guven, Phys. Rev. D57 (1998) 5158 math-ph/9804002.

[6] This assumption is not satisfied e.g. for a superconducting string; see I. Moss and S. Poletti, Phys. Lett. B199 (1987) 34 .
[7] This is obvious for $r_{c}=0$. For $r_{c} \neq 0$ one has to use also equations (2) at the brane position to obtain incompatibility (see equation (20)).

[8] Note that the vector $\square_{h} x^{\mu}+\Gamma_{\nu \lambda}^{\mu} h^{\nu \lambda}$ is normal to the brane, so the last expression of the Nambu-Goto equation with one free index $\mu$ is equivalent to the previous one with one free index $\alpha$.

[9] C.J.S. Clarke, G.F.R. Ellis and J.A. Vickers, Class. Quant. Grav. 7 (1990) 1; W.G. Unruh, G. Hayward, W. Israel and D. McManus, Phys. Rev. Lett. 62 (1989) 2897.

[10] A similar result is implicit in B. Linet, J. Math. Phys. 27 (1986) 1817.

[11] A. Vilenkin, Phys. Rev. D23 (1981) 852.

[12] In G. Kofinas, Phys. Lett. B633 (2006) 141 hep-th/0506035, the characteristic of obtaining a solution with the deficit angle remaining an integration constant has also appeared in the standard context of considering variations with respect to the brane metric for the six-dimensional Gauss-Bonnet scenario. 
[13] J. Gott, Astrophys. J. 288 (1985) 422; W. Hiscock, Phys. Rev. D31 (1985) 3288; B. Linet, Gen. Rel. Grav. 17 (1985) 1109; D. Garfinkle, Phys. Rev. D32 (1985) 1323; V.P. Frolov, W. Israel and W.G. Unruh, Phys. Rev. D39 (1989) 1084.

[14] K.P. Tod, Class. Quant. Grav. 11 (1994) 1331; J. Maluf and A. Kneip, J. Math. Phys. 38 (1997) 458 gr-qc/9505008.

[15] Fitting performed with the help of L. Perivolaropoulos.

[16] P. Bostock, R. Gregory, I. Navarro and J. Santiago, Phys. Rev. Lett. 92 (2004) 221601 hep-th/0311074; C. Charmousis and R. Zegers, JHEP 0508 (2005) 075 hep-th/0502170. 